\title{
Revision of the barley $W R K Y$ gene family phylogeny and expression analysis of the candidate genes in response to drought
}

\author{
B. YAZDANI ${ }^{1,2}$, S. SANJARI ${ }^{2}$, R. ASGHARI-ZAKARIA ${ }^{1 *}$, F. GHANEGOLMOHAMMADI ${ }^{3}$, \\ E. POURABED ${ }^{2}$, M. SHAHBAZI ${ }^{4}$, and Z.-S. SHOBBAR ${ }^{2 *}$ \\ Department of Agronomy and Plant Breeding, Faculty of Agriculture and Natural Resources, \\ University of Mohaghegh Ardabili, Ardabili 56199-11367, Iran ${ }^{1}$ \\ Department of Systems Biology, Agricultural Biotechnology Research Institute of Iran, Agricultural Research, \\ Education and Extension Organization, Karaj 31359-33151, Iran ${ }^{2}$ \\ Department of Integrated Biosciences, Graduate School of Frontier Sciences, \\ The University of Tokyo, Kashiwa, Chiba Prefecture 277-8562, Japan ${ }^{3}$ \\ Gorgan University of Agricultural Sciences and Natural Resources, Gorgan 49138-15739, Iran ${ }^{4}$
}

\begin{abstract}
The WRKY belongs to an important plant specific transcription factor families which are involved in response to various environmental stresses, as well as in growth and developmental processes. In the present report, a genome-wide identification and characterization of WRKY gene family in barley led to revision of HvWRKYs to 93 members. The phylogenetic tree was also reconstructed based on the full-length WRKY protein sequences in barley and Arabidopsis. HvWRKYs were classified into three major groups (I, II, and III) and group II was further divided to 5 subgroups (a to e). HvWRKYs were named after this classification. Interestingly, some specific motifs were discovered in subgroups IIa, IIb, and III. Analyzing the available microarray data revealed eight candidate $W R K Y$ genes which were up-regulated under drought and salinity stresses compared to the optimum conditions at seedling stage in barley. Expression profiles of these $W R K Y$ genes were validated by quantitative real-time PCR. All the investigated candidate genes (HvWRKY IIc2, HvWRKY IIIII, HvWRKY_ IIb2, HvWRKY IId4, HvWRKY III23, HvWRKY IIa5 and HvWRKY IIc19) except for $\bar{H} v W R K Y$ I8 were significantly upregulated by drought stress at the seedling stage in drought-tolerant genotype, indicating their role in drought tolerance. We hope the presented information would be helpful toward achieving drought tolerant cultivars through genetic engineering or molecular breeding.
\end{abstract}

Additional key words: Arabidopsis thaliana, chromosomal location, drought tolerance, Hordeum vulgare.

\section{Introduction}

Putative transcription factor $(T F)$ genes constitute $7 \%$ of all plant genes. TFs are proteins which play a major role in gene expression regulation (Cowell et al. 1992). When these specific proteins bind to the upstream of transcription start site (gene regulatory regions), the transcription activity will be affected (Ryu et al. 2007). The WRKY family is an outstanding higher plant TF type, which has not been found in both fungal and animal genomes (Zhang and Wang 2005, Rushton et al. 2010). Based on the phylogenetic tree classification, Magnoliophyta have more WRKY members compared to Pinophyta, Pteropsida, and Bryophyta. These TFs play a very important role in the regulation of Magnoliophyta gene expression (Berri et al. 2009). Phylogenetic analysis of WRKY family in Arabidopsis thaliana and Oryza sativa genomes has modified initial classification (Wu et al. 2005, Xie et al. 2005, Zhang and Wang 2005).

Based on the number of WRKY domain (WD) sequences (tryptophan-arginine-lysine-tyrosine) and the identity of the zinc-finger motif, the WRKY genes are classified into three main groups (I, II, and III). Furthermore, group II was divided into five subgroups (IIa, IIb, IIc, IId, and IIe). All WRKY proteins have one (groups II and III) or two (group I) conserved WDs which have a signature peptide

Submitted 5 May 2019, last revision 6 August 2019, accepted 21 August 2019.

Abbreviations: ABA - abscisic acid; TF - Transcription factor; WD - WRKY domain (sequence tryptophan-arginine-lysine-tyrosine. Acknowledgements: This work was supported by the Agricultural Biotechnology Research Institute of Iran under project number 910019151-0551-05-1. The authors are grateful to Mr. Mohammad Jedari for creating the artworks. The first two authors contributed equally to the article.

* Corresponding author; e-mail: shobbar@abrii.ac.ir, r-asghari@uma.ac.ir 
sequence of WRKYGQK at the N-terminus. Also, a $\mathrm{C}_{2} \mathrm{H}_{2-}$ (groups I and II) or a $\mathrm{C}_{2} \mathrm{HC}$-type zinc-finger motif (group III) at the C-terminus, with a total length of approximately 60 amino acids (Eulgem et al. 2000). Variants of the WRKYGQK signature motif comprise WRKYGEK and WRKYGKK, with several atypical WRKY motifs suggesting the consensus $\mathrm{W}(\mathrm{R} / \mathrm{K})(\mathrm{K} / \mathrm{R}) \mathrm{Y}$ (Xie et al. 2005). Most WRKY proteins bind preferentially to the W-box DNA sequence -(C/T)TGAC(T/C)- (Rushton et al. 1995, De Pater et al. 1996, Wang et al. 1998, Eulgem et al. 1999). Nevertheless, some WD sequences can present different binding preferences such as binding with either the SURE (sugar-responsive cis-element) or the W-box (Sun et al. 2003, Van Verk et al. 2008). Invariable WRKY amino acids signature as well as cysteine and histidine residues of the WD, which tetrahedral coordinate a zinc atom, are necessary for W-box dependent binding activity (Maeo et al. 2001).

While WRKY TFs have been implicated in some developmental processes (Johnson et al. 2002, Lagac and Matton 2004, Miao et al. 2004), their key functions are defense responses against biotic and abiotic stresses (Johnson et al. 2002, Zhou et al. 2008, Jiang and Deyholos 2009, Pandey and Somssich 2009, Wu et al. 2009, Prabu et al. 2011). Most of the genes in group IIa and IId are involved in biotic and abiotic responses. Genes in group I play a role in developmental processes and biotic and abiotic stress responses in crops, while the functions of most genes in group III should be characterized (Chen et al. 2017). In addition, all WRKY subfamilies have drought and salt stress responsive members. Also, genes in group IIc might be the crucial TFs involved in drought and salt stress responses in crops (Chen et al. 2017). Some $W R K Y$ genes in several plant species are highly expressed during seed development stage (Bakshi and Oelmüller 2014). Previous studies have suggested that WRKY TFs have major roles in pathogen-associated molecular pattern (PAMP)-triggered immunity) (PTI) and effector-triggered immunity (ETI), which are important innate immune systems in the plant (Pandey and Somssich 2009). In response to stress, WRKY TFs are involved in ABA mediated signaling pathway (Jiang et al. 2017). Members of WRKY TFs family have been identified in some plants including 92 TaWRKY in Triticum aestivum (Zhu et al. 2013), 99 OsWRKY in Oryza sativa (Ross et al. 2007), and 72 AtWRKY in Arabidopsis thaliana (Wu et al. 2005).

Barley (Hordeum vulgare L.) is a major food source with harvestable yields in harsh environments and one of the main cereal crops in developing countries. It has the highest drought stress tolerance among other crops including rice, maize and wheat, and can considerably adapt to various environmental conditions (Ullrich 2011). The barley genome (Morex genotype) has been completely sequenced (Consortium 2012), and this fact provides an excellent opportunity to raise research on barley. In the present study, we attempted to recognize members of WRKY TFs family, and their characteristics in barley via in silico approaches. We also conducted gene expression analysis of their candidate genes involved in drought stress tolerance in barley.

\section{Materials and methods}

Identification of $\boldsymbol{W R K} \boldsymbol{Y}$ gene family members in barley: Barley (126) and Arabidopsis (72) TF sequences were downloaded from the plant TFs database (PlantTFDB $v$. 4.0; http://planttfdb.cbi.edu.cn/; Jin et al. 2017). WRKY proteins from each Arabidopsis subfamily were used as a query for $t B L A S T n$ analysis in the international barley sequencing consortium (IBSC) IPK BLAST server (http://webblast.ipk-gatersleben.de/barley/) (Consortium 2012) against full length cDNA, high confidence (HC) and low confidence (LC) databases. Further, all sequences with a unigene accession number (60 unigene) for barley WRKY proteins were downloaded from the National Center for Biotechnology Information (NCBI) database (http://www. ncbi.nlm.nih.gov), as well. Additionally, hidden Markov model (HMM) profiles of WD (PF03106) were obtained from Pfam database (http://pfam.xfam.org/; Finn et al. 2016) and used to find putative WRKY proteins against barley_HighConf_genes_MIPS_23Mar12_ProteinSeq and barley_LowConf_genes_MIPS_23Mar12_ProteinSeq (Consortium 2012) using $\bar{H} M M E \bar{R}$ v. $3.1 b \bar{l}$ where the e-value threshold was set at 1 . Finally, redundant sequences were removed manually. To find all the $H v W R K Y$ transcript sequences, cDNA and protein sequences were used as queries for BLASTn and BLASTp searches against Ensembl plants database (IBSC_v. 2; http://plants.ensembl.org/ index.html), respectively. All non-redundant sequences were checked in the Pfam database for existence of WD.

Chromosomal Location of HvWRKYs on barley genome: The cDNA and protein sequences were employed as the queries for BLASTn and $t B L A S T n$ searches against EnsemblPlants database (http://plants.ensembl.org/index. html) to find the location of HvWRKY genes on barley chromosomes, respectively. The distribution of $H v W R K Y \mathrm{~s}$ was created using the MapChart v. 2.3 software (Voorrips 1994).

Phylogenetic analysis: Multiple sequence alignment (MSA) of the WRKY transcription factors for fulllength protein sequences from Arabidopsis and barley were obtained using ClustalX program (Larkin et al. 2007). Phylogenetic tree was constructed using the neighbor-joining (NJ) method by MEGA7 (http://www. megasoftware.net/; Kumar et al. 2006), and 1000 boot $^{-}$ strapping replicates. The Gonnet matrix method was used to compute the evolutionary distances and estimate the number of amino acid substitutions per site.

Detection of additional conserved motifs of WRKY proteins: Recognition of the HvWRKY protein motifs was performed using motif elicitation tool (MEME; v. 4.11.4; http://meme-suite.org/tools/meme; Bailey et al. 2009). The setting options included any number of repetitions, ten and sixty amino acids for the minimum and maximum width of a motif, respectively, and 15 for the maximum number of motifs to find. All discovered motifs by $M E M E$ were scanned in InterPro database (http://www.ebi.ac.uk/ interpro/; Bailey et al. 2009). 
In silico WRKY gene expression survey: Microarray data, $22 \mathrm{~K}$ Affymetrix gene chip array (Barley 1), were downloaded from GEO (http://www.ncbi.nlm.nih.gov/ gds/) containing accession numbers GSE3170, GSE6990, GSE15970, and GSE17669 for drought, plus GSE3097 and GSE5605 for salinity. Based on the annotation results, 22 probes were detected for HvWRKYs in the Affymetrix barley 1 chip. Affymetrix raw data [cell intensity (CEL)] files were used as input files in Expression Console software (v. 1.3.1.187). The raw data was normalized using robust multi-array analysis (RMA) through sketch quantile normalization approach (Irizarry et al. 2003). Then, the Flexarray software package (v. 1.6.1) was employed to statistically analyze the normalized array data via analysis of variance (ANOVA). A hierarchical cluster map was created (Pearson method) to find the differentially expressed genes using online heat mapper (http://www. heatmapper.ca/expression/).

Plants and drought stress treatments: Seeds of spring barley (Hordeum vulgare L.) cv. Yousef (a drought-tolerant Iran native cultivar) and Morocco 9-75 (a droughtsusceptible international cultivar) were obtained from the Seed and Plant Improvement Institute (SPII) and the International Center for Agricultural Research in the Dry Area (ICARDA), respectively (Ceccarelli 1994, Abedini et al. 2017). The plant growth and water treatment were done as previously described (Abedini et al. 2017). Briefly, the culture was performed in a greenhouse located at the Agricultural Biotechnology Research Institute of Iran (ABRII), Karaj, Iran (a 12-h photoperiod with an average photosynthetic active radiation density of $180 \mu \mathrm{mol} \mathrm{m}^{-2} \mathrm{~s}^{-1}$ and day/night temperatures of $23 / 20^{\circ} \mathrm{C}$ ), as a complete random design (CRD) with three biological replications (a replication included a pot containing 6 plants). Two levels of water irrigation including 70 and $10 \%$ of water holding capacity of soil were considered as optimum and severe drought stress conditions, respectively. The samples were obtained from young fully expanded leaves of both 36-day-old genotypes grown under optimum conditions or a gradual drought stress for 21 days. Then, leaves were rapidly dipped into liquid nitrogen and stored at $-80{ }^{\circ} \mathrm{C}$ until RNA extraction.

Expression study of $\mathrm{HvWRKYs}$ genes under drought stress: Total RNAs were isolated using Trizol reagent (Invitrogen, Carlsbad, USA) and were treated with RNasefree DNase (Geneall, Seoul, Korea) according to the manufacturer's instruction. RNA samples were reverse transcribed to cDNA with oligo $(\mathrm{dT})_{18}$ and random primers using cDNA synthesis kit (Takara, Tokyo, Japan).

Gene-specific primers for eight $H v W R K Y$ genes (HvWRKY IIc2, HvWRKY IIIII, HvWRKY IIb2, HvWRKY IId4, HvWRKY III23, HvWRKY I8, HvWRKY_IIa5, and HvWRKY_IIc19) were designed using Oligo software (Rychlik 2010 ) that are described in Table 1 Suppl1. Real time qRT-PCR was performed on a LightCycler ${ }^{\circledR 96}$ (Roche, Mannheim, Germany) using SYBR Green Supermix (Takara). The reactions were performed using the following program: $95^{\circ} \mathrm{C}$ for
5 min and 40 cycles $\left(95{ }^{\circ} \mathrm{C}\right.$ for $30 \mathrm{~s}, 58^{\circ}$ or $61^{\circ} \mathrm{C}$ for $30 \mathrm{~s}$ and $72{ }^{\circ} \mathrm{C}$ for $30 \mathrm{~s}$ ). Barley Actin gene was used as the internal control (Tommasini et al. 2008). Each reaction was carried out in three independent biological replicates with the relative expression was calculated using the $2^{-\Delta \Delta \mathrm{CT}}$ method (Schmittgen and Livak 2008) by REST 2009 software (Pfaffl et al. 2009). Gene expression in drought-sensitive genotype under optimum condition was used as a calibrator for calculating the relative gene expression. The relative gene expressions of candidate genes were analyzed by a complete random design where the average values of treatments were calculated by the least significant difference (LSD) test using $S A S$ statistical package $(P<0.01)$ (Afifi et al. 2003).

\section{Results and discussion}

In this study, 93 WRKY gene loci were discovered encoding 497 proteins in Hordeum vulgare. In the first study of $W R K Y$ gene family in barley, 46 members were recognized and named from $H v W R K Y 1$ to $H v W R K Y 46$ (Mangelsen et al. 2008). Then, fifteen novel HvWRKY members, HvWRKY57 to HvWRKY61, were found (Meng and Wise 2012). A recent study in barley discovered 34 additional WRKY TFs from HvWRKY62 to HvWRKY95 (Liu et al. 2014). Notably, previous studies revealed that $H v W R K Y 1$ was also published as HvWRKY38 (Eckey et al. 2004, Mangelsen et al. 2008). Further, HvWRKY 56 was the full-length protein of HvWRKY8 (Liu et al. 2014).

Our investigation revealed that HvWRKY35 (DQ863119) and HvWRKY41 (AK365469/DQ863124) reported in the previous studies belong to the same unigene accession (Hv.9019). According to the results, HvWRKY72 (MLOC 3853) includes the complete protein and coding sequence version of HvWRKY49 (JQ806390) and, the genes are mapped to the same locus (HORVU3Hr1G081000) on the barley genome. Although, there are different unigene accessions for HvWRKY3 (AK359706) and HvWRKY52 (AK368514); (Hv.13567 and Hv.32682, respectively), the protein and coding sequence alignments indicated that they are different transcripts of HORVU5Hr1G065420. In addition, HvWRKY31 (DQ863115/MLOC 5971) and HvWRKY95 (MLOC_15430) were completely similar to different transcripts of HORVU1Hr1G069620. We also found three novel WRKY TFs. The additional information on HvWRKY TFs including previous and new WRKY names, chromosomal locations, unigenes, cDNA, and protein accession numbers, Ensembl and MIPS accession numbers, number of the introns, as well as the protein length and position of WD have been provided in Table 1 Suppl2.

The full length protein sequences of WRKY TFs in barley and Arabidopsis were aligned and the unrooted phylogenetic tree was constructed. Based on the number of WRKY domains, the sequence similarity of both WRKY domain and zinc-finger motif, as well as on the phylogenetic tree, HvWRKYs were classified into three main groups (I, II, III) which is in accordance to previous reports in barley 


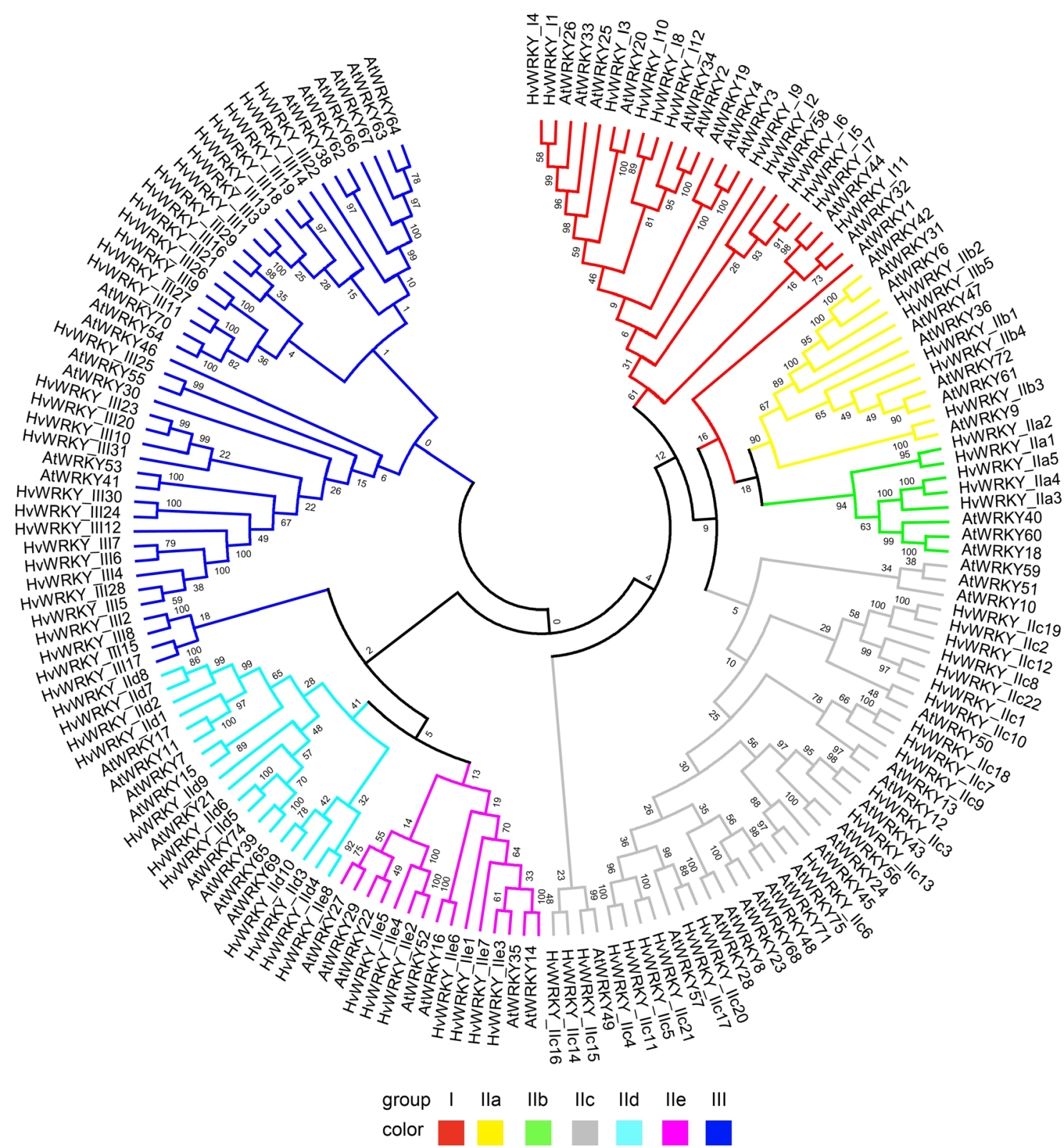

Fig. 1. An unrooted phylogenetic tree of WRKY proteins from barley (HvWRKY) and Arabidopsis (AtWRKY). Protein sequences were aligned using ClustalX and the unrooted phylogenetic tree was constructed using MEGA7 by the neighbor-joining method with 1000 bootstraps. The WRKY proteins were classified into 7 subgroups (I, IIa, IIb, IIc, IId, IIe, and III).

Chromosomal locations of 10 genes (HvWRKYII1, HvWRKY_IIb5, HvWRKY_IIc19, HvWRKY_IIc20, HvWRKY_IIc21, HvWRKY IIc22, HvWRKY III28, HvWRKY III29, HvWRKY III30, and HvWR KY III31) were indistinct on the barley genome. However, the rest of HvWRKY genes (83 out of 93) were physically mappéd on barley chromosomes according to the Ensembl database (Fig. 2). The HvWRKY members were distributed on all chromosomes of barley, whereas chromosome 3 contained the maximum number of $H v W R K Y \mathrm{~s}$, having 21 of them (23\%), followed by $17 H v W R K Y \mathrm{~s}$ (18\%) on chromosome 5, and 5 HvWRKYs (5\%) on chromosome 6.

and other plants (Eulgem et al. 2000, Mangelsen et al. 2008, Meng and Wise 2012, Liu et al. 2014). Furthermore, group II was divided into five (a, b, c, d, and e) subgroups, respectively. In barley, the zinc finger motif in group II was similar to group I, with the CX4-5CX22-23HXH pattern, while the zinc finger in group III had CX7CX23HXC pattern based on WRKY domain alignment (Fig. 1 Suppl.). $H v W R K Y$ genes were given a generic name based on their classification (HvWRKY I, HvWRKY IIa, HvWRKY IIb, HvWRKY_IIc, HvWRKY IId, HvWRKY IIe, HvWRKY III) with an additional number (e.g., 1, 2,1...) and also decimal for different transcripts encoded by the same locus (e.g., HvWRKY I1.1- HvWRKY I1.2) (Table 1 Supp12.).

Comparison between the unrooted phylogenetic tree in this study and the preceding report by Liu et al. (2014) revealed that all the members were placed in the same 
subgroups except four members (i.e. HvWRKY IId3, HvWRKY IId4, HvWRKY III20, and HvWRKY III29). HvWRKY IId3 and HvWRKY IId4 belonged to the IIe in the aforementioned report while they were clustered into IId subgroup in this study. These members joined AtWRKY65 which has classified in IIe or IId subgroups according to two previous studies (Wu et al. 2005, He et al. 2012). Anyway, IId and IIe subgroups always cluster into a single clade. In addition, HvWRKY III20 and HvWRKY III29 were located in subgroup III $\overline{\text { in the }}$ present study while they were classified in IIe and IIc in the former report, respectively (Liu et al. 2014). AtWRKY41, AtWRKY 53, AtWRKY54, AtWRKY55, and AtWRKY70 belonging to the group III based on the phylogenetic tree of Arabidopsis (Wu et al. 2005) were joined to the HvWRKY III20 and HvWRKY III29.

Group $\overline{\mathrm{I}}$ contained 12 and $1 \overline{3}$ WRKYs in barley and Arabidopsis, respectively (Fig. 1). AtWRKY1 joined to group II in Arabidopsis and barley uprooted phylogenetic tree but it has two WRKY domains thereby was classified into group I. All members in group I had two WRKY domains except for HvWRKY I10 with one WRKY domain. This phenomenon was observed in foxtail millet (Zhang et al. 2017). Also, 6 out of 31 WRKY members belonging to group I in radish had one WD (Karanja et al. 2017). This suggests that they might have either experienced domain loss or gain events during evolution (Ross et al. 2007). According to the studies on unicellular green alga Chlamydomonas reinhardtii with a great genetic similarity with plant ancestors, there is only one $W R K Y$ gene which is a part of group I (with two WDs). This can indicate that group I is the actual ancestor of $W R K Y$ family from which other groups of the family have been evolved (Goel et al. 2016). Searching in GeneBank revealed that there are WRKY genes which are similar to group I in two non- photosynthetic eukaryotic organisms: one in Dictyostelium discoideum (AAO52331) and the other one in a protozoan called Giardia lamblia (EAA40901). This generally confirms the hypothesis of group I as being the true ancestor of WRKY genes. WRKY genes originated about one and a half to two billion years from early eukaryotes, prior to their transfer into the plant kingdom. However, there is no logical explanation behind their expansion into plants kingdom while being absent in yeasts and animals (Ülker and Somssich 2004).

Group II was classified into five subgroups in barley: IIa, IIb, IIc, IId, and IIe (Fig. 1) as in the classification of WRKY family in wheat, rice, and Arabidopsis (Wu et al. 2005, Rushton et al. 2010, Zhu et al. 2013). Subgroup IIa, IIb, IIc, IId, and IIe contained 5, 5, 22, 10, and 8 members, respectively. Similar to Jatropha curcas, 10 out of 12 members in group II in barley had an extra plant zinc domain (pfam10533) upstream of the WRKY (Xiong et al. 2013). In other studies, the evolution of group II from group I have been acknowledged given the loss of amino terminal WD. The results also suggested that group II has been evolved from the carboxyl terminal WD of group I (Zhang and Wang 2005). In proteins of group I, carboxyl domain and amino domain help DNA-binding activities and specialization of the bind, respectively. So, because of the transcription factor DNA-binding nature, the evolution of group II from carboxyl domain seems to be logical.

On average, about $20 \%$ of $W R K Y$ gene family in higher plants belongs to group III while the members of this group do not exist in moss (Physcomitrella patens). This indicates that group III of WRKY genes has evolved following groups I and II (Dong et al. 2003, Kalde et al. 2003) and exists only in higher plants. Interestingly, HvWRKY_III24 and HvWRKY_III30 had two WDs, but they were classified in group III given the zinc finger motif pattern (C2-H2). $33 \%$ (31 members) where $18 \%$ WRKY members in barley and Arabidopsis belonged to group III, respectively (Fig. 1). Compared to Arabidopsis, group III in rice was far more active in terms of evolution since in the sequences of the group III of rice, additional duplication has been observed (Eulgem et al. 2000) with $34 \%$ of $W R K Y$ genes falling into group III in rice (Ross et al. 2007). Hence, the diversity in the number of WRKY members in group III was represented by an expansion of WRKY members of this group in barley. Alignment of multiple HvWRKY domain amino acid sequences (Fig S1) showed group I and II had histidine (H) in the conserved domain whereas group III had cysteine (C). Not only, HvWRKYIII_2, HvWRKYIII_8, HvWRKYIII 15, and HvWRKYIII_17 followed group III conserved domain pattern, but also they shared same amino acid motifs pattern according to the Fig. 2 Suppl. Therefore, these genes were classified into group III.

The additional conserved motifs (15 putative motifs) were discovered by $M E M E$ program using the longest proteins transcript of all members of WRKY subfamily in barley. Typically, most WRKY members within the same cluster in phylogenetic tree shared the same motif compositions. Therefore, these WRKY proteins have similar functions (Xie et al. 2018). Predictably, most of the HvWRKYs in the same subgroups had similar motif arrangements (Fig. 2 Suppl.). Therefore, the reliability of the grouping in barley was supported by phylogenetic analysis results along with the similarities of conserved motif compositions in each subgroup. The motif 1 and motif 9 contained conserved WRKYGQK domain where at least a number of them were present in all barley WRKY amino acid sequences. The motif 7 consisted of plant zinc domain which only existed in most of the subgroup IId members. On the other hand, biological functions of other motifs were unknown by InterPro database scanning (Table 2 Suppl1.). Furthermore, motif 8 and motif 12 were observed in members of subgroups IIa and IIb. Also, motif 5 and 10 were only found in some of the members in group III. These specific motifs seem to be important in the functional divergence of $W R K Y$ genes.

To recognize differentially expressed $H v W R K Y s$ under drought stress conditions at seedling and reproductive stages, four microarray datasets (GSE3170, GSE6990, GSE17669, and GSE15970) were investigated. Evaluating gene expression in barley seedlings of cv. Morex under different soil water content (SWC) (GSE6990) revealed that HvWRKY_IIc2, HvWRKY_IIb2, HvWRKY_III23, $H v W R K Y \_I I a 5$, and $H v W R K Y \_I I c \overline{1} 9$ were significantly upregulated under low soil water content. Further, $H v W R K Y$ 


\section{Chr1}

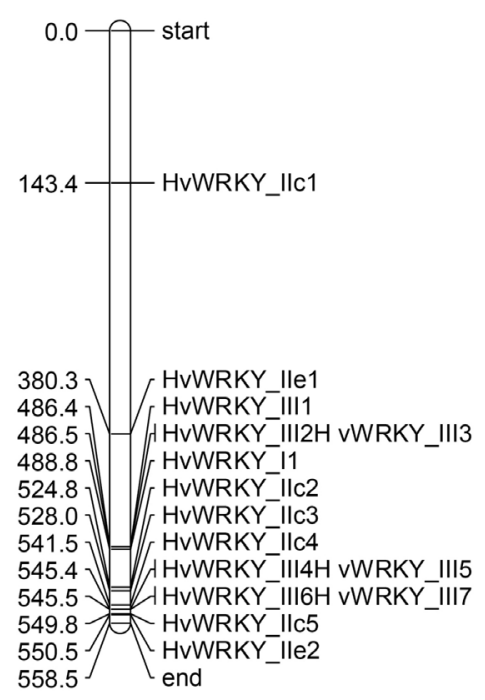

Chr2

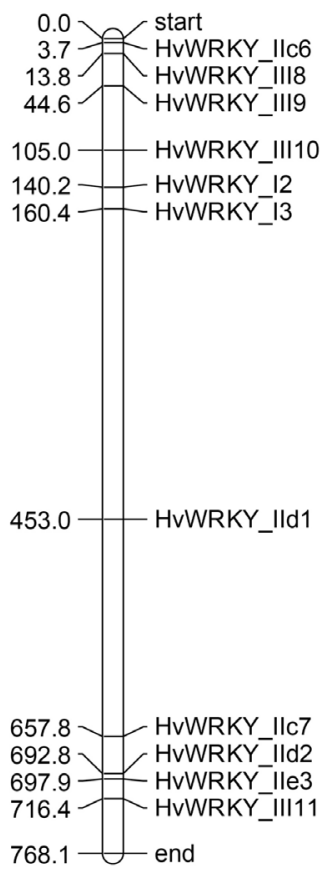

Chr3

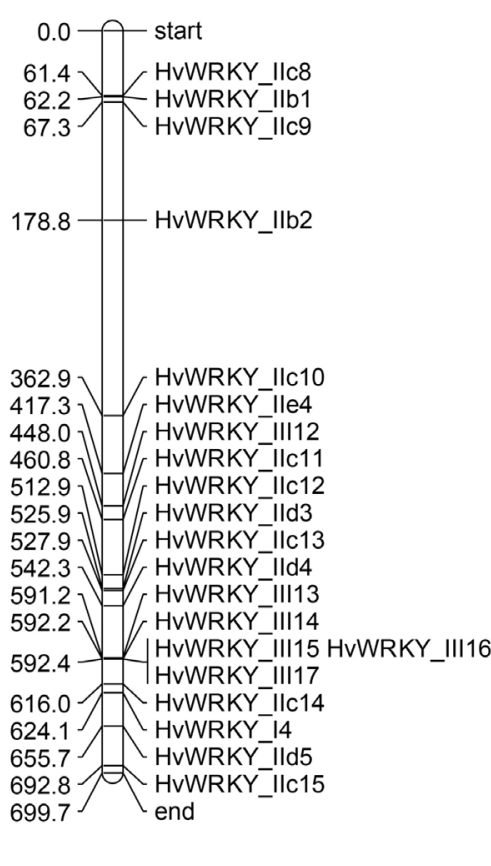

\section{Chr4}

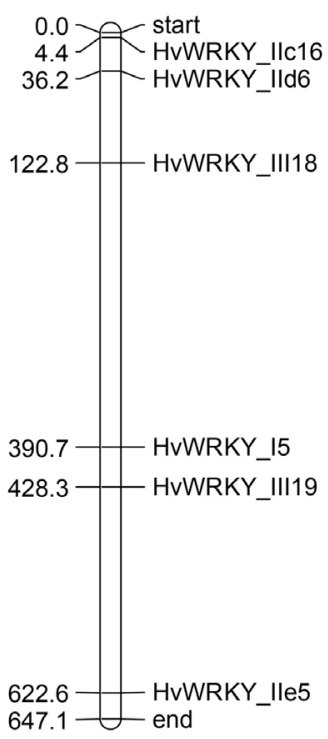

Chr5

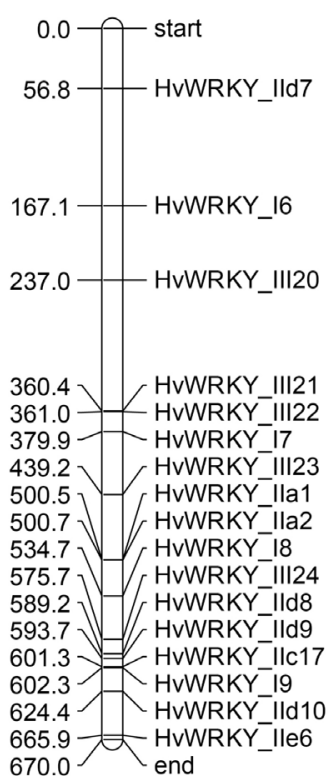

Chr6

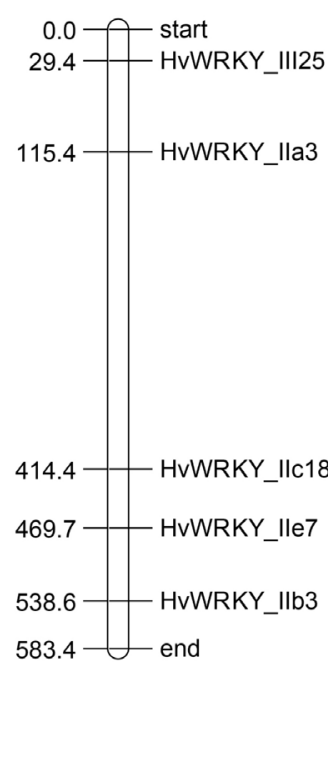

\section{Chr7}

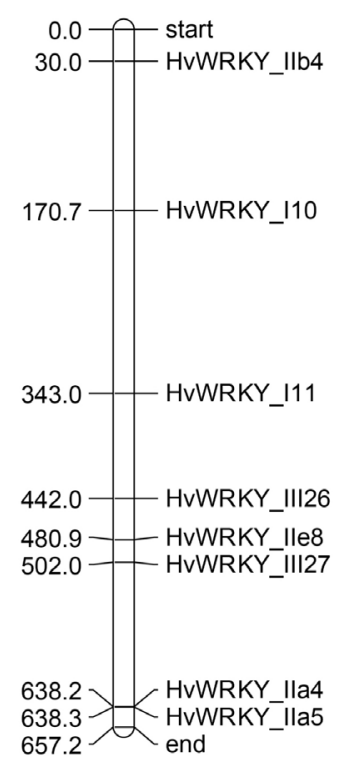

Fig. 2. Distribution of $H v W R K Y$ genes (83 out of 93) on the barley chromosomes (chromosomal distances are given in megabases). Chromosomal locations of $10 H v W R K Y$ members are uncertain on the barley genome.

IIb2 gene expression was up-regulated more when soil moisture declined gradually (Fig. 3). Comparisons between drought stress and control conditions at seedling stage in cv. Oregon Wolfe dominant and recessive genotypes indicated that the transcription of HvWRKY_I8 and HvWRKY_IIc19 were elevated under drought stress (Fig. 3). Differential gene expression analysis in two drought-tolerant and a drought-sensitive genotypes at reproductive stage under drought stress revealed that $H v W R K Y$ IIe 3 was highly expressed in both drought-tolerant genotypes. Furthermore, the transcription of HvWRKY_IIII1 and HvWRKY III7 grew in only one drought-tolerant genotype (Fig. 3). Finally, assessing differential gene expression on the photosynthetic organs of the barley spike (lemma, palea, and awn) and seed between drought and control conditions indicated that $H v W R K Y$ IIb2 was the only up-regulated 


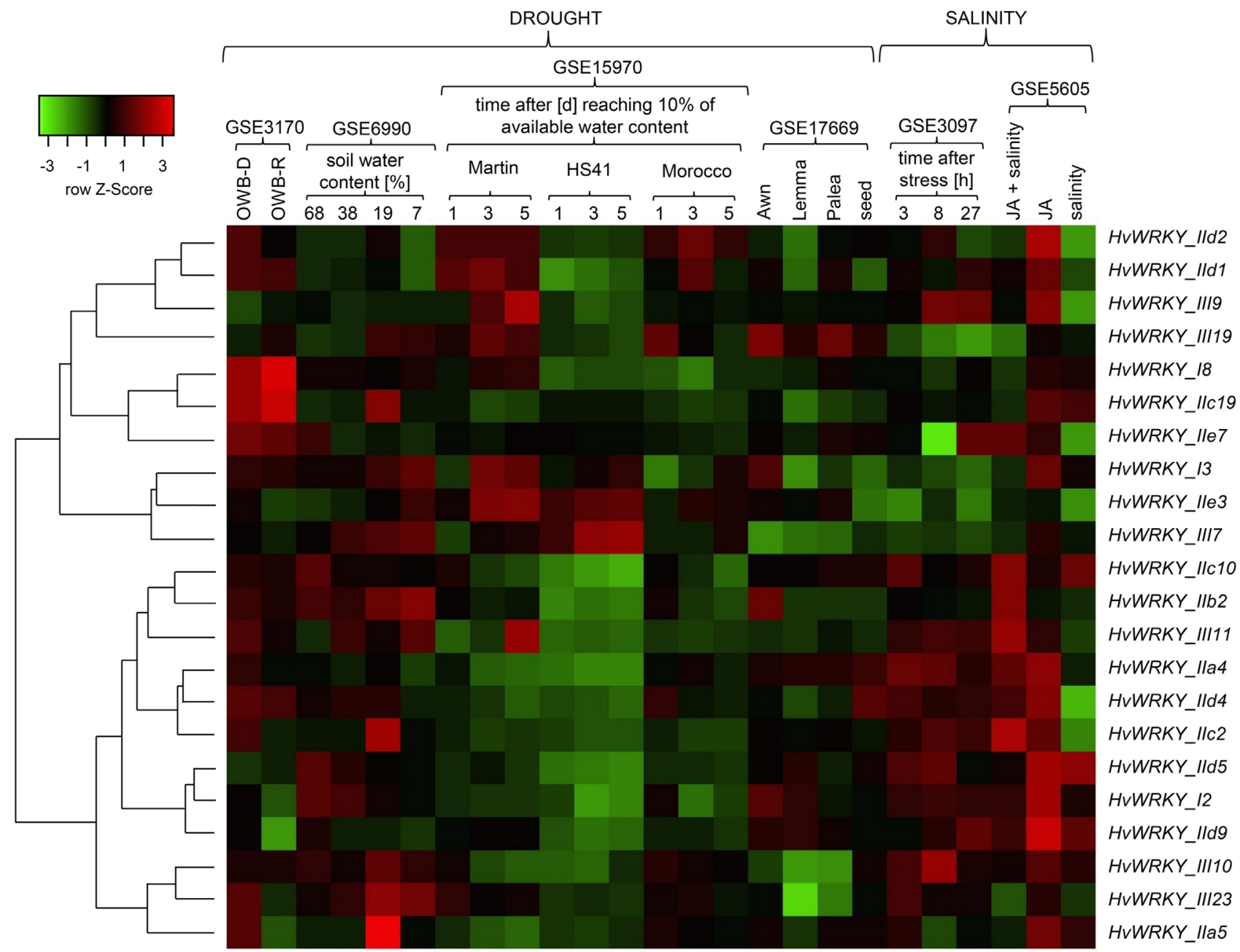

Fig. 3. Hierarchical clustering differential expression profiles of barley WRKY genes in 25 samples under drought and salinity stresses compared to optimum conditions.

member in the awn under drought (Fig. 3). Thus, it seems that HvWRKY_IIc2, HvWRKY_IIb2, HvWRKY_III23, HvWRKY I8, $\bar{H} v W R K Y$ IIa5, and HvWRKY IIc $\overline{1} 9$ are involved in the response to drought stress at the seedling stage. On the other hand, HvWRKY_IIIT, HvWRKY_IIe3, and $H v W R K Y$ IIb2 play a role in response to drought stress at the reproductive stage.

To investigate the $H v W R K Y$ gene expression patterns under salinity stress, three microarray datasets were considered. Studying differential gene expression at seedling stage under control conditions and salinity (GSE3097) with three time points indicated that $H v W R K Y$ IIIIO was up-regulated under salinity (Fig. 3). According to a previous report (Walia et al. 2007), two kinds of genes with specific gene expression patterns would be good candidate genes to enhance the salinity tolerance: 1 ) genes with down-regulated expression under salinity stress while altered in pre-treatment with jasmonic acid (JA) followed by salinity; 2) genes which were up-regulated in JA pretreatment followed by salinity stress which also responded to salinity and JA treatments as well. Therefore, we predict that HvWRKY_IIc2, HvWRKY_IIII1, HvWRKY_IIb2, and HvWRKY IId4 may enhance salinity tolerance at the seedling stage (GSE5605) based on their gene expression patterns (Fig. 3).

Some HvWRKY genes (HvWRKY IIc2, $H v W R K Y$ IIb2, HvWRKY III23, HvWRKY I8, HvWRKY IIa5, and $H v W R K Y$ IIc1 $\overline{\overline{9}}$ ) were selected based on the aforementioned microarray datasets analysis to confirm the gene expression patterns at seedling stage in droughtsensitive and tolerant genotypes under drought conditions by real-time PCR. Drought and salt stresses both cause cellular dehydration. Further, their signal transduction pathways have cross talk with each other (Bartels and Sunkar 2005, Miller et al. 2010). HvWRKY IIIII and $H v W R K Y \_$IId 4 were significantly up-regulated in response to salinity via JA pathway. We hypothesized that they may play a role in response to drought stress; thus, we selected them for further investigation under drought conditions as well.

The phylogenetic analysis revealed that one (HvWRKYI8), one (HvWRKY IIa5), one (HvWRKY IIb2), two (HvWRKY IIc2 and HvWRKY IIc19), one (HvWRKY_IId4), and two (HvWRKY_IIIII and HvWRKY 


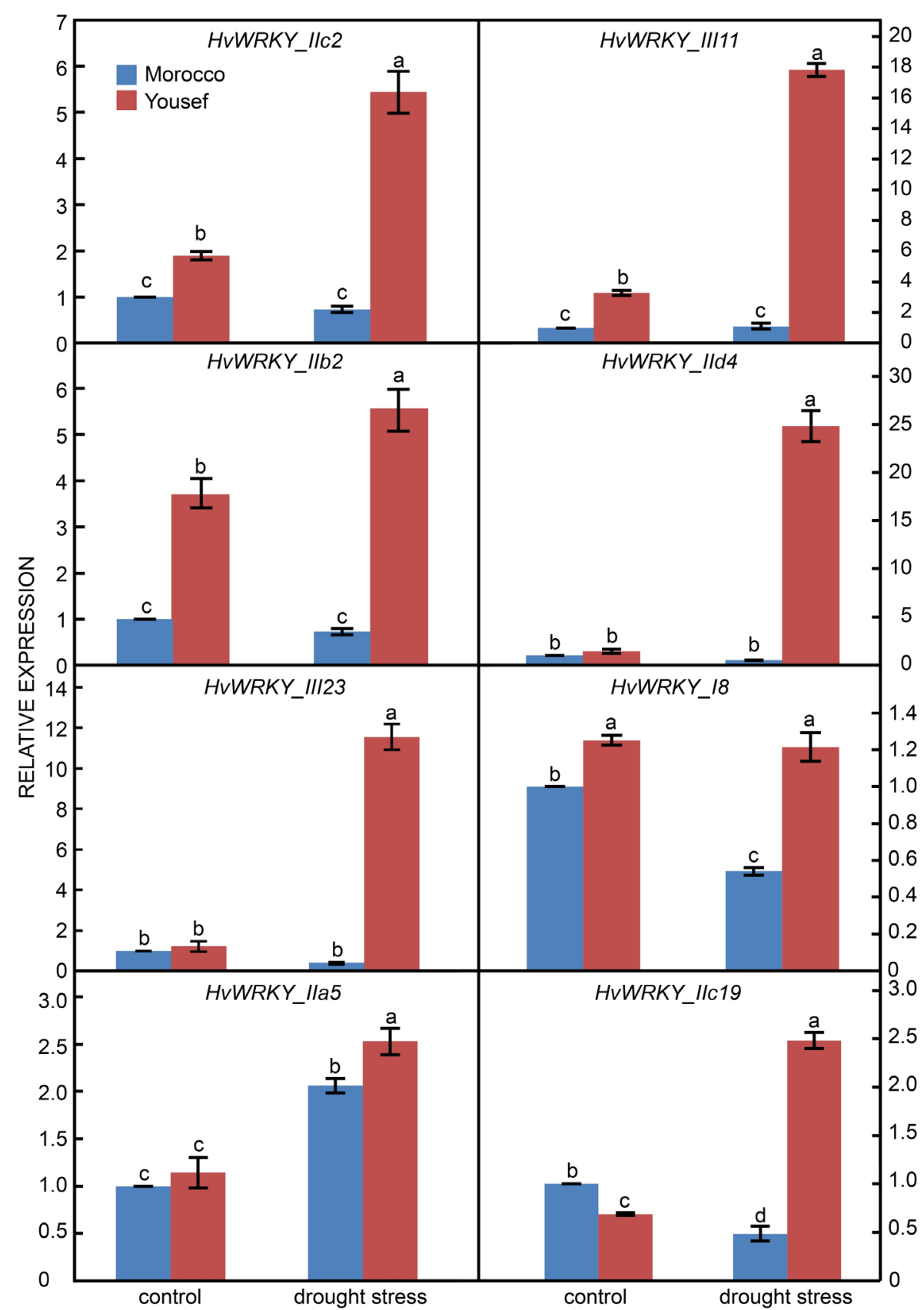

Fig. 4. Relative expression profiles of eight $H v W R K Y$ genes at the seedling stage with three independent biological replicates in drought-tolerant (Yousef) and drought-sensitive (Morocco) cultivars of barley under severe drought stress. Transcription in the sensitive genotype under water optimum conditions was used as a calibrator. $Y$-axis and error bars represent the fold change and standard deviation, respectively.

III23) candidate genes belong to the group I, IIa, IIb, IIc, IId, and III cluster, respectively (Fig. 2). Analysis of variance indicated that the effects of water treatments were significant on the relative expression of all eight $H v W R K Y$ genes $(P \leq 0.01)$ under both optimum and drought stress conditions (Table 3 Suppl1.). The expression of $H v W R K Y$ IIc2, HvWRKY IIIII, HvWRKY IIb2, HvWRKY IId $\overline{4}$, and $H v W R K Y \overline{I I I} 23$ was significantly up-regulated in the tolerant cultivar (Yousef) while their expressions remained unchanged in the sensitive genotype (Morocco) under drought stress. HvWRKY IIa5 was significantly upregulated in both genotypes under drought stress at the seedling stage though more in Yousef than in Morocco. HvWRKY IIc19 was notably up-regulated in the tolerant (Yousef) genotype under severe drought stress, while it was down-regulated in the susceptible Morocco genotype. No significant change was found in the transcription of HvWRKY I8 in Yousef, while it was reduced in Morocco under drought stress at the seedling stage (Fig. 4).

Based on the phylogenetic tree, HvWRKY IIc2 and 
HvWRKY IIc19 were closely joined together, while $H v W R K Y$ IIa5 gene was clustered with HvWRKY IIa4, HvWRKY IIa3, AtWRKY18, and AtWRKY60 (Fig. 2). A previous report indicated that HvWRKY IIc19 (HvvWRKY19) and HvWRKY IIa4 (HvvWRKY2) are upregulated in vegetative organs of the resistant genotype but not in the sensitive genotype of barley under drought (Li et al. 2014). Also, HvWRKY IIa3 (HvWRKY1/38) is involved in response to abiotic stress including cold and drought stress at the seedling stage in barley via an ABA-independent way (Mar et al. 2004). AtWRKY18 and AtWRKY60 in the cluster are transcriptional activators in response to ABA and abiotic stresses such as drought and salinity in Arabidopsis wild type (Chen et al. 2010). Based on the obtained gene expression results (Fig. 4), the aforementioned microarray data (Fig. 3), and phylogenetic clustering (Fig. 2), HvWRKY IIa5 and HvWRKY IIc19 seem to be the transcriptional activator in response to drought-stress.

HvWRKY IIII1 along with the HvWRKY III27, AtWRKY54, and AtWRKY70 lie in the same group based on the phylogenetic tree (Fig. 2). The wrky54/wrky70 double mutant reveals earlier leaf senescence compared to wild type in Arabidopsis. Thus, these two genes cooperate with each other and they are negative regulators in senescence (Besseau et al. 2012). In addition, AtWRKY46, AtWRKY54, and AtWRKY70 are related to OsWRKY47 in rice. Knock-out and overexpression of OsWRKY47 lead to drought susceptibility and tolerance, respectively (Raineri et al. 2015). Overall, the obtained results suggest that $H v W R K Y$ IIIII might act as a negative regulator of leaf senescence, helping the genotype to survive under drought conditions.

\section{Conclusions}

In the present study, 93 members of $W R K Y$ gene family were identified in barley, classified into three groups and seven subgroups based on unrooted phylogenic tree in barley and Arabidopsis. Eight drought-responsive candidate WRKY genes were predicted based on analysis of available microarray datasets under drought and salinity at the seedling stage in barley. Furthermore, the gene expression patterns in tolerant and susceptible genotypes by qRT-PCR indicated that all of candidate genes $(H \nu W R K Y$ IIc2, HvWRKY IIIII, HvWRKY IIb2, HvWRKY IId $\overline{4}$, HvWRKY III23, HvWRKY IIa5, and HvWRKY IIc19) are involved in the response to the drought stress at the seedling stage in barley. The achieved results provided useful information regarding the HvWRKY gene family and its drought-responsive members for further functional analysis. They might also be beneficial for achieving drought tolerant cultivars through molecular breeding or genetic engineering.

\section{References}

Abedini, R., Ghane Golmohammadi, F., Pishkam Rad, R., Pourabe,d E., Jafarnezhad, A., Shobbar, Z.-S., Shahbazi, M.: Plant dehydrins: shedding light on structure and expression patterns of dehydrin gene family in barley. - J. Plant Res. 130:747-763, 2017.

Afifi, A., May, S., Clark, V.: Computer-aided Multivariate Analysis. - CRC Press, Boca Raton 2003.

Bailey, T.L., Boden, M., Buske, F.A., Frith, M., Grant, C.E., Clementi, L., Ren, J., Li, W.W., Noble, W.S.: MEME SUITE: tools for motif discovery and searching. - Nucl. Acids Res. 37:202-208, 2009.

Bakshi, M., Oelmüller, R.: WRKY transcription factors. - Plant Signal. Behav. 9: e27700, 2014.

Bartels, D., Sunkar, R.: Drought and salt tolerance in plants. Plant Sci. 24: 23-58, 2005.

Berri, S., Abbruscato, P., Faivre-Rampant, O., Brasileiro, A.C., Fumasoni, I., Satoh, K., Kikuchi, S., Mizzi, L., Morandini, P., Pè, M., Piffanelli, P.: Characterization of WRKY coregulatory networks in rice and Arabidopsis. - BMC Plant Biol. 9: 120, 2009.

Besseau, S., Li, J., Palva, E.T.: WRKY54 and WRKY70 co-operate as negative regulators of leaf senescence in Arabidopsis thaliana. - J. exp. Bot. 63: 2667-2679, 2012.

Ceccarelli, S.: Specific adaptation and breeding for marginal conditions. - Euphytica 77: 205-219, 1994.

Chen, F., Hu, Y., Vannozzi, A., Wu, K., Cai, H., Qin, Y., Mullis, A., Lin, Z., Zhang, L.: The WRKY transcription factor family in model plants and crops. - Plant Sci. 36: 311-335, 2017.

Chen, H., Lai, Z., Shi, J., Xiao, Y., Chen, Z., Xu, X.: Roles of Arabidopsis WRKY18, WRKY40 and WRKY60 transcription factors in plant responses to abscisic acid and abiotic stress. BMC Plant Biol. 10: 281, 2010.

Consortium, T.I.B.G.S.A.: Physical, genetic and functional sequence assembly of the barley genome. - Nature 491: 711716, 2012.

Cowell, I.G., Skinner, A., Hurst, H.C.: Transcriptional repression by a novel member of the bZIP family of transcription factors. - Mol. cell. Biol. 12: 3070-3077, 1992.

De Pater, S., Greco, V., Pham, K., Memelink, J., Kijne, J.: Characterization of a zinc-dependent transcriptional activator from Arabidopsis. - Nucl. Acids Res. 24: 4624-4631, 1996.

Dong, J., Chen, C., Chen, Z.: Expression profiles of the Arabidopsis $W R K Y$ gene superfamily during plant defense response. - Plant mol. Biol. 51: 21-37, 2003.

Eckey, C., Korell, M., Leib, K., Biedenkopf, D., Jansen, C., Langen, G., Kogel, K.H.: Identification of powdery mildew induced barley genes by cDNA-AFLP: functional assessment of an early expressed MAP kinase. -Plant mol Biol. 55: 1-15, 2004.

Eulgem, T., Rushton, P.J. Robatzek, S., Somssich, I.E.: The WRKY superfamily of plant transcription factors. - Trends Plant Sci. 5: 199-206, 2000.

Eulgem, T., Rushton, P.J. , Schmelzer, E., Hahlbrock, K., Somssich, I.E.: Early nuclear events in plant defence signalling: rapid gene activation by WRKY transcription factors. - EMBO J. 18: 4689-4699, 1999.

Finn, R.D., Coggill, P., Eberhardt, R.Y., Eddy, S.R., Mistry, J., Mitchell, A.L., Potter, S.C., Punta, M., Qureshi, M., Sangrador-Vegas, A., Salazar, G.A., Tate, J., Bateman, A.: The Pfam protein families database: towards a more sustainable future. - Nucl.Acids Res. 44: 27-285, 2016.

Goel, R., Pandey, A. Trivedi, P.K. , Asif, M.H.: Genome-wide analysis of the Musa WRKY gene family: evolution and differential expression during development and stress. - Front. 
Plant Sci. 7: 299, 2016.

He, H., Dong, Q., Shao, Y., Jiang, H., Zhu, S., Cheng, B., Xiang, Y.: Genome-wide survey and characterization of the WRKY gene family in Populus trichocarpa. - Plant Cell Rep. 31: 1199-1217, 2012.

Irizarry, R.A., Hobbs, B., Collin, F., Beazer-Barclay, Y.D., Antonellis, K.J., Scherf, U., Speed, T.P.: Exploration, normalization, and summaries of high density oligonucleotide array probe level data. - Biostatistics 4: 249-264, 2003.

Jiang, J., Ma, S., Ye, N., Jiang, M., Cao, J. Zhang, J.: WRKY transcription factors in plant responses to stresses. - J. integr. Plant Biol. 59: 86-101, 2017.

Jiang, Y., Deyholos, M.K.: Functional characterization of Arabidopsis NaCl-inducible WRKY25 and WRKY33 transcription factors in abiotic stresses. - Plant mol. Biol. 69: 91-105, 2009.

Jin, J., Tian, F., Yang, D.C., Meng, Y.Q., Kong, L., Luo, J., Gao, G.: PlantTFDB 4.0: toward a central hub for transcription factors and regulatory interactions in plants. - Nucl. Acids Res. 45: 1040-1045, 2017.

Johnson, C. S., Kolevski, B., Smyth, D.R.: TRANSPARENT TESTA GLABRA2, a trichome and seed coat development gene of Arabidopsis, encodes a WRKY transcription factor. Plant Cell 14: 135-1375, 2002.

Kalde, M., Barth, M., Somssich, I.E., Lippok, B.: Members of the Arabidopsis WRKY Group III transcription factors are part of different plant defense signaling pathways. - Mol. Plant-Microbe Interact. 16: 295-305, 2003.

Karanja, B.K., Fan, L., Xu, L., Wang, Y., Zhu, X., Tang, M., Wang, R., Zhang, F., Muleke, E.M., Liu, L.: Genome-wide characterization of the WRKY gene family in radish (Raphanus sativus L.) reveals its critical functions under different abiotic stresses. - Plant Cell Rep. 36:1757-1773, 2017.

Kumar, S., Nei, M., Dudley, J., Tamura, K.: MEGA: A biologistcentric software for evolutionary analysis of DNA and protein sequences. - Brief. Bioinformatics 9: 299-306, 2008.

Lagac, M., Matton, D.P.: Characterization of a WRKY transcription factor expressed in late torpedo-stage embryos of Solanum chacoense. - Planta 219: 185-189, 2004.

Larkin, M.A., Blackshields, G., Brown, N.P., Chenna, R., Mcgettigan, P.A., McWilliam, H., Valentin, F., Wallace, I.M., Wilm, A., Lopez, R., Thompson, J.D., Gibson, T.J., Higgins, D.G.: Clustal W and Clustal X version 2.0. - Bioinformatics 23: 2947-2948, 2007.

Li, H., Guo, Q., Lan, X., Zhou, Q., Wei, N.: Comparative expression analysis of five $W R K Y$ genes from Tibetan hulless barley under various abiotic stresses between droughtresistant and sensitive genotype. - Acta Physiol. Plant. 36: 963-973, 2014.

Liu, D., Leib, K., Zhao, P., Kogel, K.H., Langen, G.: Phylogenetic analysis of barley WRKY proteins and characterization of HvWRKY1 and -2 as repressors of the pathogen-inducible gene HvGER4c. - Mol. Genet. Genomics 289: 1331-1345, 2014.

Maeo, K., Hayashi, S., Kojima-Suzuk, H., Morikami, A., Nakamura, K.: Role of conserved residues of the WRKY domain in the DNA-binding of tobacco WRKY family proteins. - Biochemistry 65: 2428-2436, 2001.

Mangelsen, E., Kilian, J., Berendzen, K.W., Kolukisaoglu, U.H., Harter, K., Jansson, C., Wanke, D.: Phylogenetic and comparative gene expression analysis of barley (Hordeum vulgare) WRKY transcription factor family reveals putatively retained functions between monocots and dicots. - BMC Genomics 9: 194, 2008.

Mar, C., Mazzucotelli, E., Crosatti, C., Francia, E., MichelStanca, A., Cattivelli, L.: Hv-WRKY38: a new transcription factor involved in cold- and drought-response in barley. Plant mol. Biol. 55: 399-416, 2004.

Meng, Y., Wise, R.P.: HvWRKY10, HvWRKY19, and HvWRKY28 regulate Mla-triggered immunity and basal defense to barley powdery mildew. - Mol. Plant-Microbe Interact. 25: 1492-1505, 2012.

Miao, Y., Laun, T., Zimmermann, P., Zentgraf, U.: Targets of the WRKY53 transcription factor and its role during leaf senescence in Arabidopsis. - Plant mol. Biol. 55: 853-867, 2004.

Miller, G., Suzuki, N., Ciftci-Yilmaz, S., Mittler, R.: Reactive oxygen species homeostasis and signalling during drought and salinity stresses. - Plant Cell Environ. 33: 453-467, 2010.

Pandey, S.P., Somssich, I.E.: The role of WRKY transcription factors in plant immunity. - Plant Physiol. 150: 1648-1655, 2009.

Pfaffl, M.: REST 2009 Software User Guide. - Qiagen, Hilden 2009.

Prabu, G., Kawar, P.G., Pagariya, M.C., Prasad, D.T. Identification of water deficit stress upregulated genes in sugarcane. - Plant Mol. Biol. Rep. 29: 291-304, 2011.

Raineri, J., Wang, S., Peleg, Z., Blumwald, E., Chan, R.L.: The rice transcription factor OsWRKY47 is a positive regulator of the response to water deficit stress. - Plant mol. Biol. 88: 401-413, 2015.

Ross, C.A., Liu, Y., Shen, Q.J.: The WRKY gene family in rice (Oryza sativa). - J. integr. Plant Biol. 49: 827-842, 2007.

Rushton, P.J., Macdonald, H., Huttly, A.K., Lazarus, C.M., Hooley, R.: Members of a new family of DNA-binding proteins bind to a conserved cis-element in the promoters of Amy2 genes. - Plant mol. Biol. 29: 691-702, 1995.

Rushton, P.J., Somssich, I.E., Ringler, P., Shen, Q.J.: WRKY transcription factors. - Trends Plant Sci. 15: 247-258, 2010.

Rychlik, W.: PCR primer design. In: Yuryev, A. (ed.): OLIGO 7 primer analysis software. Pp. 35-59. Humana Press, Totowa 2007.

Ryu, T., Jung, J., Lee, S., Nam, H.J., Hong, S.W., Yoo, J.W., Lee, D.K., Lee, D.: bZIPDB: A database of regulatory information for human bZIP transcription factors. - BMC Genomics. 8: 136, 2007.

Schmittgen, T. D., Livak, K. J.: Analyzing real-time PCR data by the comparative CT method. - Nat. Protoc. 3:1101-1108, 2008.

Sun, C., Palmqvist, S., Olsson, H., Borén, M., Ahlandsberg, S., Jansson, C.: A novel WRKY transcription factor, SUSIBA2, participates in sugar signaling in barley by binding to the sugar-responsive elements of the iso1 promoter. - Plant Cell 15: 2076-2092, 2003.

Tommasini, L., Svensson, J.T., Rodriguez, E.M., Wahid, A., Malatrasi, M., Kato, K., Wanamaker, S., Resnik, J., Close, T.J.: Dehydrin gene expression provides an indicator of low temperature and drought stress: transcriptome-based analysis of barley (Hordeum vulgare L.). - Funct. Integr. Genomics 8: 387-405, 2008.

Ülker, B., Somssich, I.E.: WRKY transcription factors: from DNA binding towards biological function. - Curr. Opin. Plant Biol. 7: 491-498, 2004.

Ullrich, S.E.: Barley: Production, Improvement, and Uses. Wiley-Blackwell, Ames 2011.

Van Verk, M.C., Pappaioannou, D., Neeleman, L., Bol, J.F. Linthorst, H.J.M.: A novel WRKY transcription factor is required for induction of $P R-1 a$ gene expression by salicylic acid and bacterial elicitors. - Plant Physiol. 146: 1983-1995, 2008 .

Voorrips, R.E.: Computer note mapchart: software for the graphical presentation of linkage maps and QTLs. - J. Hered. 
93: 77-78, 2002.

Walia, H., Wilson, C., Condaine, P., Liu, X., Ismail, A.M., Close, T.J.: Large-scale expression profiling and physiological characterization of jasmonic acid-mediated adaptation of barley to salinity stress. - Plant Cell Environ. 30: 410-421, 2007.

Wang, Z., Yang, P., Fan, B., Chen, Z.: An oligo selection procedure for identification of sequence-specific DNA-binding activities associated with the plant defence response. - Plant J. 16: 515$522,1998$.

Wu, K.L., Guo, Z.J., Wang, H.H., Li, J.: The WRKY family of transcription factors in rice and Arabidopsis and their origins. - DNA Res. 12: 9-26, 2005

Wu, X., Shiroto, Y., Kishitani, S., Ito, Y., Toriyama, K.: Enhanced heat and drought tolerance in transgenic rice seedlings overexpressing OsWRKY11 under the control of HSP101 promoter. - Plant Cell Rep. 28: 21-30, 2009.

Xie, T., Chen, C., Li, C., Liu, J., Liu, C., He, Y.: Genome-wide investigation of $W R K Y$ gene family in pineapple: evolution and expression profiles during development and stress. BMC Genomics 19: 490, 2018.

Xie, Z., Zhang, Z.L., Zou, X., Huang, J., Ruas, P., Thompson,
D., Shen, Q. J.: Annotations and functional analyses of the rice $W R K Y$ gene superfamily reveal positive and negative regulators of abscisic acid signaling in aleurone cells. - Plant Physiol. 137: 176-189, 2005.

Xiong, W., Xu, X., Zhang, L., Wu, P., Chen, Y., Li, M., Jiang, H., Wu, G.: Genome-wide analysis of the WRKY gene family in physic nut (Jatropha curcas L.). - Gene 524: 124-132, 2013.

Zhang, L., Shu, H., Zhang, A.Y., Liu, B.L., Xing, G.F., Xue, J.A., Yuan, L.X., Gao, C.Y., Li, R.Z.: Foxtail millet WRKY genes and drought stress. - J. agr. Sci. 155: 777-790, 2017.

Zhang, Y., Wang, L.: The WRKY transcription factor superfamily: its origin in eukaryotes and expansion in plants. - BMC Evol. Biol. 5: 1, 2005.

Zhou, Q.Y., Tian, A.G., Zou, H.F., Xie, Z.M., Lei, G., Huang, J., Wang, C.M., Wang, H.W., Zhang, J.S., Chen, S.Y.: Soybean WRKY-type transcription factor genes, GmWRKY13, GmWRKY21, and GmWRKY54, confer differential tolerance to abiotic stresses in transgenic Arabidopsis plants. - Plant Biotechnol. J. 6: 486-503, 2008.

Zhu, X., Liu, S., Meng, C., Qin, L., Kong, L., Xia, G.: WRKY transcription factors in wheat and their induction by biotic and abiotic stress. - Plant mol. Biol. Rep. 31: 1053-1067, 2013. 